\title{
The role of diffusion tensor imaging in idiopathic sensorineural hearing loss: is it significant?
}

\author{
Walaa Mahmoud ${ }^{1 B, D, E}$, Wessam Elshawaf ${ }^{2 B, D}$, Ahmed Tawfik ${ }^{1 A, C, F}$ \\ 'Department of Diagnostic Radiology, Mansoura University, Egypt \\ ${ }^{2}$ Audiology Unit, ENT Department, Mansoura University, Egypt
}

\section{Abstract}

Purpose: To assess the role of diffusion tensor imaging metrics in the evaluation of the microstructural integrity of the central auditory tract in patients with idiopathic sensorineural hearing loss (SNHL), and to compare these patients with healthy controls.

Material and methods: This prospective study, which was conducted upon 30 subjects ( 21 males, 9 females; age range from 16 to 65 years, mean age 45years) with SNHL proven by audiometric tests. Ten age- and sex-matched healthy volunteers were included as a control group. Patients $(n=30)$ and volunteers $(n=10)$ underwent conventional magnetic resonance imaging and diffusion tensor imaging of the brain. Both fractional anisotropy and mean diffusivity (MD) of 3 points along the acoustic tract (inferior colliculus, lateral lemniscus and superior olivary nucleus) were measured bilaterally in all patients and correlated with controls.

Results: Mean fractional anisotropy (FA) values were reduced bilaterally at the superior olivary nucleus and/or lateral lemniscus and more significantly at the inferior colliculus of subjects with SNHL in comparison to the volunteers. In patients of unilateral SNHL, similar results were obtained in the contralateral side when compared to controls with statistically significant difference at the 3 regions $(p=0.001)$. No significant changes were noticed in the MD parameters either in patient or control groups.

Conclusions: The FA value was a valuable non-invasive biomarker in evaluating the subtle microstructural abnormalities of the central auditory tract in idiopathic SNHL and correlated well with hearing impairment.

Key words: diffusion tensor, idiopathic SNHL, central auditory pathway.

\section{Introduction}

Hearing is a complex process in which many parts of the ear contribute to transmit signals to the brain. The auditory system consists of both peripheral structures (external, middle, and inner ear) as well as central regions (cochlear nuclei in the medulla oblongata, superior olivary nuclei and lateral lemniscus in the pons, inferior colliculus in the midbrain, medial geniculate nuclei in the thalamus, and auditory cortex in the superior temporal Heschl gyrus). Most of auditory fibres undergo a contralateral decussa- tion to the opposite superior olive in the region known as the trapezoid body [1].

The primary effect of hearing loss is impaired communication, which can adversely affect a person's fruitful life and healthy social relationships [2].

The broad term "sensorineural hearing loss" (SNLH) has been used by clinicians to refer to either a malfunctioning inner ear or a retrocochlear problem affecting the canalicular VIII cranial nerve and cerebellopontine angle or that involves the higher (central) auditory nuclei and neural tracts. Identifying the aetiology of hearing loss

Correspondence address:

Dr. Walaa Mahmoud, Department of Diagnostic Radiology, Mansoura University, Egypt, e-mail: walaamahmoud@mans.edu.eg

Authors' contribution:

A Study design · B Data collection · C Statistical analysis · D Data interpretation - E Manuscript preparation · F Literature search · G Funds collection 
is valuable to establish a treatment strategy that can help to prevent or slow down complete loss of auditory function $[2,3]$.

The central auditory structures are involved in various processes that may occur in isolation from those involving peripheral receptors [4]. SNHL can be a consequence of different conditions, including viral infection, tumour, ischaemia, multiple sclerosis, or congenital malformations [5].

Despite continuous efforts to delineate the pathophysiological attributes of SNHL, the aetiology remains mostly unclear with nearly $90 \%$ of cases being idiopathic. Therefore, finding a method to accurately predict microstructural changes of the auditory circuit is extremely important $[5,6]$.

In patients with SNHL, radiological evaluation of temporal bone and retrocochlear abnormalities are typically investigated by computed tomography (CT) and/or magnetic resonance imaging (MRI). Computed tomography plays an essential role in the evaluation of bony details and when MRI is contraindicated. Although MRI is a sufficient tool to diagnose gross congenital anomalies of the inner ear and to determine the anatomy of the cochlear nerve, information about changes in the neuronal auditory circuit is limited, and subtle changes may be overlooked $[3,7,8]$.

In recent years, advanced MR-based modalities: magnetic resonance spectroscopy (MRS), MR morphometry, functional MRI, and diffusion tensor imaging, have revealed the microstructural and functional changes of the auditory system. For instance, MRS can easily show the changes of the content and concentration of some metabolites in the central auditory system. Also, functional MRI reveals the differences in the activation of the central auditory circuit in healthy and idiopathic SNHL subjects [4].

Recently, diffusion tensor imaging (DTI) has been used widely as a research tool to investigate the microstructural integrity associated with abnormalities of white matter [9-11]. Organized packed axonal tracts, overlying sheets of myelin, microtubules, and neurofilaments represent physical barriers imposing directionality of water movement in the tissues of the central nervous system [12-14] because water diffusion will be restricted perpendicular to the axis of the axons, causing anisotropy [15]. By applying diffusion-weighted imaging (DWI) in multiple directions, the tensor (which is a three-dimensional diffusion model) can be estimated and a fibre-tracking map could be generated. The diffusion tensor indices fractional anisotropy (FA) and mean diffusivity (MD) were calculated for each voxel, enabling a non-invasive and sensitive quantitative tool to delineate anisotropic diffusion $[9,10,13,16]$. Fractional anisotropy is expressed as a scalar value ranging from 0 (corresponding to unrestricted diffusion in all directions) to 1 (corresponding to unrestricted diffusion in only one direction), as in healthy brain tissues. So, declining FA is a sensitive index for loss of white matter integrity [17].
The calculated mean diffusivity (MD) gives the total magnitude of water diffusion. Changing of tissue density, damage to white matter fibres, or overlying myelin sheath will influence these indices by altering of the directionality of water motion [18].

This work aims to evaluate subtle and microstructural changes of auditory neural tracts by comparison of DTI metrics - FA and MD in idiopathic SNHL patients and normal individuals, and to correlate between FA and MD values, and hearing threshold levels in those patients.

\section{Material and methods}

\section{Patients}

The current study was approved by the Institutional Review Board (IRB) of our institute, and informed consent was obtained from each of the patients as well as controls prior to the examination. The present work comprised 40 individuals divided into a patient group and a control group. The patient group included 30 patients with SNHL (21 males, 9 females) with a mean age of 45 years, ranging from 16 to 65 years. Based on audiometric tests, 16 patients (53.33\%) had bilateral SNHL whereas 14 (46.66\%) had unilateral affection. The participants in the control group comprised 10 age-matched individuals with normal hearing ( 7 male, 3 female) with a mean age of 43.4 years, ranging from 18 to 62 years. All subjects enrolled in the current study had idiopathic SNHL with normal anatomy of peripheral and central auditory circuits in conventional MRI study. The control group underwent brain MRI for reasons other than SNHL. The participants had no history of brain surgery, trauma, infection, or ototoxic medication intake. Children who were too young were not included in our study, to avoid false positive results due to developing a myelination process. Three patients had suffered from vertigo and 4 had coexistent mastoiditis. All patients were submitted to full audiological history, otoscopic examination, and basic audiological evaluation (pure tone audiometry, speech reception thresholds, and speech discrimination tests). Hearing loss ranged from mild (from 30 to $90 \mathrm{db}$ ) to profound (hearing threshold more than $90 \mathrm{db}$ ).

\section{Magnetic resonance imaging}

All studies were acquired using a 1.5 Tesla scanner (Ingenia, Philips). A dStream HeadNeckSpine 20-channel coil was used. Before DTI acquisition a non-contrast conventional MR study was done to exclude gross abnormalities of the peripheral and central auditory pathway. The MR imaging protocols included the following sequences: 1) the T1 parameters were $\mathrm{TR}=475$ and $\mathrm{TE}=15 \mathrm{~ms}$; 2) $\mathrm{T} 2$ parameters were $\mathrm{TR}=3626$ and $\mathrm{TE}=100 \mathrm{~ms}$; and 3 ) FLAIR parameters were $\mathrm{TR}=6000, \mathrm{TE}=120$, and $\mathrm{T}=2800 \mathrm{~ms}$ with $\mathrm{FOV}=230$ $\times 177 \mathrm{~mm}^{2}$, matrix $=80 \times 80$, and slice thickness $=5 \mathrm{~mm}$. 


\section{Diffusion tensor imaging}

DTI was acquired in the axial plane using a standard singleshot, echo-planar imaging pulse sequence with parallel imaging. Imaging parameters were TR of 1463, TE of $83 \mathrm{~ms}$, FOV of $224 \times 224 \mathrm{~mm}^{2}$ and a data matrix of $92 \times 88$, leading to voxel dimensions $\left(2.43 \times 2.54 \times 2.5 \mathrm{~mm}^{3}\right)$. Diffusion-encoding gradients were applied along $32 \mathrm{di}$ rections, using a minimum $b$-value of 0 and maximum $b$-value of $1000 \mathrm{~s} / \mathrm{mm}^{2}$. Slice thickness was $2.5 \mathrm{~mm}$ with gap of 0 , and with the total acquisition time of about 7-8 min.

\section{Image assessment}

The DICOM image processing was performed on extended MR Workspace 2.6.3.5 workstation, Philips Medical Systems, Nederland B.V - supplied by the vendor. Firstly, automated registration of the DTI data was done to eliminate distortion artifacts, then quantitative analysis of FA and MD were calculated for each patient and control.

Two-pixel seed 3 ROIs within the acoustic circuit (superior olivary nucleus, the lateral lemniscus, the inferior colliculus) were traced bilaterally by a neuroradiologist. As showed in Figure 1, ROI selection was carried out by using of color-encoded FA map. Diffusion tensor metrics were measured bilaterally at the three regions and compared to controls. In patients with unilateral SNHL, the contralateral measurements were compared to controls and free ipsilateral side.

\section{Statistical analysis and data interpretation}

Data were analysed using IBM SPSS software package version 22.0. Qualitative data were described using the number and percentage. Quantitative data were described by calculating the means and standard deviations for parametric data after testing normality using the KolmogorovSmirnov test with $p$-values $<0.05$ considered as statistically significant. Student's $t$-test was used to compare FA and MD values in 2 independent groups (patients and controls) at 3 anatomical sites.

The Pearson product-moment correlation was used to determine the correlation between changes in the FA measures and hearing loss level in the patient group.

The diagnostic accuracy of a test was measured using receiver operating characteristic (ROC) curve analysis to discriminate diseased cases from non-diseased cases. Sensitivity and specificity were detected from the curve and PPV; NPV and accuracy were calculated through cross tabulation.

\section{Results}

The study included 30 patients with idiopathic SNHL. The affection was bilateral in 53\% $(n=16)$ and unilateral in $47 \%(n=14)$.
No significant difference in sex distribution between patient and control groups with control group displayed slightly lower mean age than patients group yet with no statistical significance.

All patient underwent conventional MR imaging before DTI was obtained and all of them showed normal imaging findings except four patients who had coexistent mastoiditis.

Mean values of FA and MD measured in the ipsilateral tract in patients with unilateral SNHL and controls showed no statistical differences in the 3 regions $(p>0.05)$, although 4 patients with profound unilateral SNHL showed reduced FA values when compared to corresponding values in controls.

Diffusion anisotropy evaluated with measured FA was found to be reduced in at least 1 of 3 ROIs on the central acoustic tract (superior olivary nucleus, lateral lemniscus, and inferior colliculus) in all patients.

Table 1 summarizes the mean and standard deviation of FA and MD values measured in 3 regions in the acoustic tract in both patients and control groups.

The mean FA values were $0.288 \pm 0.029$ and $0.391 \pm$ 0.049 at the superior olivary nucleus in patients and controls, respectively, with significant statistical difference $(p=0.001)$. The mean MD values were $0.737 \pm 0.071$ and $0.743 \pm 0.058 \times 10^{-3} \mathrm{~mm}^{2} / \mathrm{s}$ in patients and controls, respectively, with no statistical difference $(p=0.76)$.

The mean FA values were $0.497 \pm 0.04$ and $0.601 \pm 0.04$ at the lateral lemniscus in patients and controls, respectively, with significant statistical difference $(p=0.001)$. Mean MD values of the lateral lemniscus were $0.765 \pm 0.04$ and $0.785 \pm 0.05 \times 10^{-3} \mathrm{~mm}^{2} / \mathrm{s}$ in patients and controls, respectively, with no statistical difference $(p=0.11)$.

The mean FA values were $0.680 \pm 0.047$ and 0.803 \pm 0.03 at the inferior colliculus in patients and controls, respectively, with significant statistical difference $(p=0.001)$. The mean MD values of inferior colliculus were 0.695 \pm 0.054 and $0.699 \pm 0.049 \times 10^{-3} \mathrm{~mm}^{2} / \mathrm{s}$ in patients and controls, respectively, with no statistical difference $(p=0.72)$.

The difference between mean FA values in patients and control groups was higher at the inferior colliculus than at the lateral lemniscus and superior olivary nucleus. The best result obtained with area under curve (AUC) of FA at the inferior colliculus was 0.987. Selection of 0.763 as a cut-off point to differentiate between both groups revealed an accuracy of $96.7 \%$, sensitivity of $95.0 \%$, and specificity of $96.1 \%$ (Figure 2).

There was a negative correlation between FA values and age of the patients more pronounced at inferior colliculus region.

Correlations between FA and MD in the 3 regions and measured hearing levels are summarized in Table 2. An inverse relationship existed between FA and the degree of hearing loss, which was more significant at the inferior colliculus ( $p=0.001$ and $r=-0.949$ ), whereas no significant correlation occurred between MD and the severity of hearing impairment. 

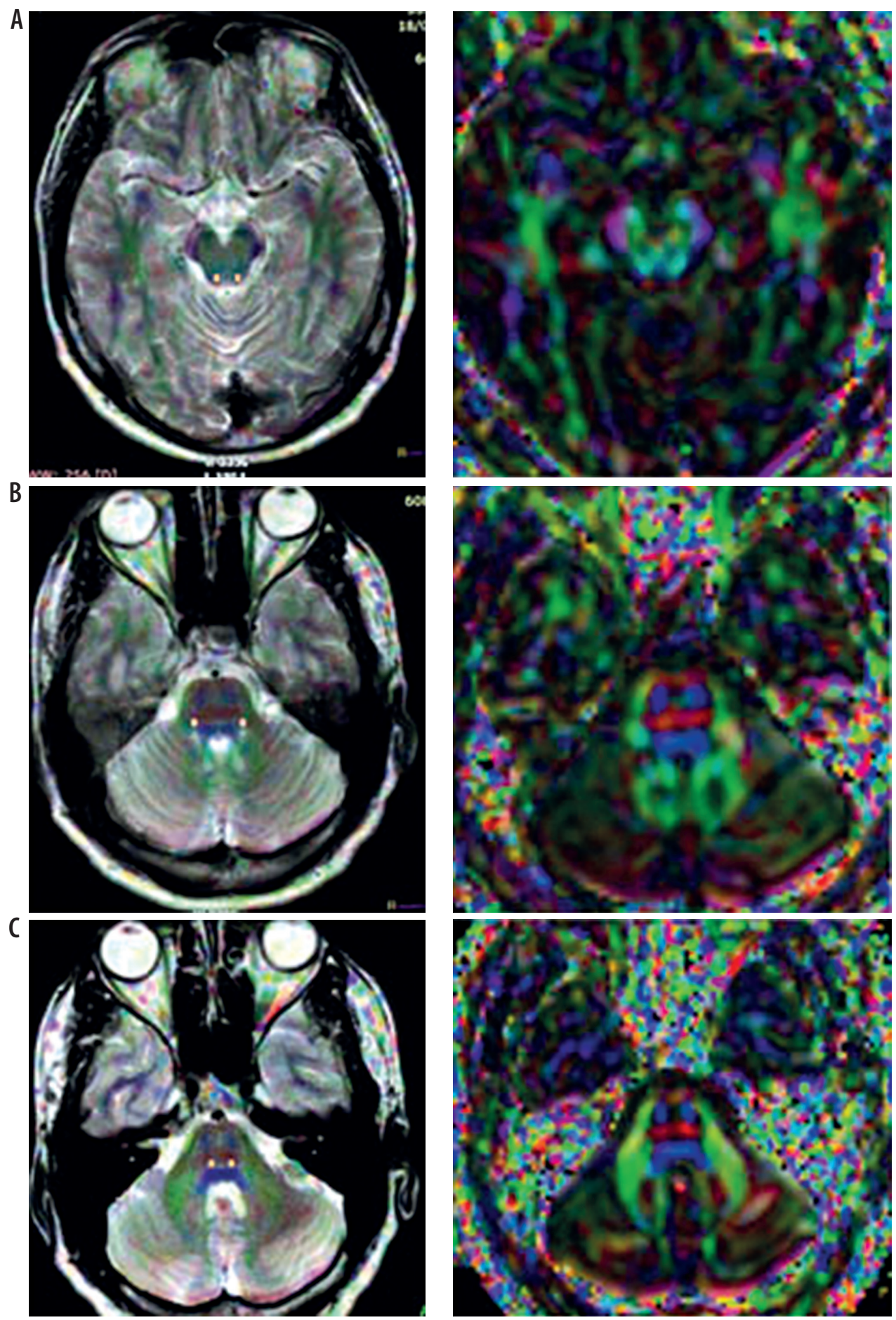

Figure 1. ROI was drawn bilaterally in the inferior colliculus (A), lateral lemniscus (B), and superior olivary nucleus (C) in a colour-coded fractional anisotropy map and FA map

\section{Discussion}

Sensorineural hearing loss is a very common complaint in otological and audiological practices. For about $7 \%$ of patients, a defined cause can be recognized and a specific regimen used for treatment. The remaining patients show no detectable cause of hearing loss despite adequate investigations and are categorized as "idiopathic" [19]. Immediate and efficient recognition and treatment of idiopathic SNHL can improve hearing recovery and life traits $[20,21]$.
There is limited knowledge about the changes occurring in the central acoustic pathways with SNHL. Although conventional MRI is considered indispensable in the assessment of SNHL, it is relatively insufficient in the evaluation of microstructure and dysfunction of white matter. Previous studies reported that there was a reduction in the volume of cortical grey matter, and brain activities were diminished in various cortical and subcortical areas, including the inferior colliculus and temporal gyrus [22-25]. DTI research in the field of neurology has shown 
Table 1. Mean and standard deviation of fractional anisotropy (FA) and mean diffusivity (MD) values measured at 3 regions in the acoustic tract in patients and control groups

\begin{tabular}{|l|l|c|c|c|c|}
\hline \multicolumn{2}{|l|}{} & Control & Cases & Student's $t$ test & $p$-value \\
\hline \multirow{2}{*}{ Lateral meniscus } & FA & $0.601 \pm 0.04$ & $0.485 \pm 0.04$ & 11.44 & $<0.001^{*}$ \\
\cline { 2 - 6 } & MD & $0.785 \pm 0.05$ & $0.765 \pm 0.04$ & 1.64 & 0.11 \\
\hline Inferior colliculus & FA & $0.806 \pm 0.03$ & $0.680 \pm 0.047$ & 11.77 & $<0.001^{*}$ \\
\cline { 2 - 6 } & MD & $0.699 \pm 0.049$ & $0.695 \pm 0.054$ & 0.36 & 0.72 \\
\hline Superior olivary nucleus & FA & $0.391 \pm 0.049$ & $0.288 \pm 0.029$ & 9.32 & $<0.001^{*}$ \\
\cline { 2 - 6 } & MD & $0.743 \pm 0.058$ & $0.737 \pm 0.071$ & 0.31 & 0.76 \\
\hline
\end{tabular}

Cases group: patients with bilateral + affected side of unilateral sensorineural hearing loss. Control group: control individuals + normal side in unilateral SNHL patients

promising results that indicate the usefulness of this novel radiologic approach $[26,27]$. In the context of auditory disorders, diffusion tensor metrics can give quantitative data about the myelination state and microstructural integrity of the central auditory circuit and provide valuable prognostic information about clinical outcomes if cochlear implantation is considered $[7,18]$.

In previous studies, regions related to the primary auditory cortical structures such as the gyrus of Heschl, the superior temporal gyrus, and the auditory radiation were the most frequently imaged regions.

Both the inferior colliculus and lateral lemniscus were less commonly examined [7]. In the current study, 3 ROI were selected bilaterally in the superior olivary nucleus, lateral lemniscus, and inferior colliculus to obtain DTI metrics. In their study, Lin et al. 2008 focused on DTI measurements at only the lateral lemniscus and the inferior colliculus. The 2 previously mentioned regions were favoured because they are more easily distinguished in the auditory tract. Additionally, the auditory fibres in these areas are mainly vertically oriented, and this facilitates measurements of the metrics with the guidance of colourencoded FA maps. However, other areas are less discernible because they contain a lace of longitudinal, transverse, and oblique fibres, and so the reconstructed images might suffer from a poor spatial resolution and higher signal-tonoise ratio [28].

Because of the decussation of the auditory neural fibres in a trapezoid body, central activities were decreased markedly in the contralateral auditory pathway cranial to the cochlear nucleus [25].

Sensorineural hearing loss can cause cortical and subcortical microstructural changes in several auditory neurological structures because the development of cortical myelination is essentially dependent on a variety of sound stimuli in pre- and post-lingual deaf [7,25,28-30]. In the same context, Emmorey et al. found that acoustic deprivation caused poor myelination $[25,31]$.

Activity-dependent enhancement in the thickness and abundance of myelin has been traced in several central neuronal structures of vertebrates, not only in development but also in maturity [32-36].
Table 2. Correlation analysis between fractional anisotropy (FA), mean diffusivity (MD), and severity of sensorineural hearing impairment

\begin{tabular}{|l|c|c|}
\multirow{3}{*}{ FA: superior olive } & $r$ & -0.496 \\
\cline { 2 - 3 } & $p$ & 0.041 \\
\hline MD: superior olive & $r$ & 0.021 \\
\cline { 2 - 3 } & $p$ & 0.943 \\
\hline FA: lateral meniscus & $r$ & $-0.650^{*}$ \\
\cline { 2 - 3 } & $p$ & 0.012 \\
\hline FD: lateral meniscus & $r$ & -0.327 \\
\cline { 2 - 3 } & $p$ & 0.254 \\
\hline MD: inferior colliculus & $r$ & $-0.949^{* *}$ \\
\cline { 2 - 3 } & $p$ & $<0.001$ \\
\hline
\end{tabular}

Hearing level measured using a calibrated pure tone audiometer.

The main finding in our study was reduced mean FA values bilaterally in patients with bilateral SNHL and on the contralateral side in patients with unilateral SNHL, when compared to healthy controls. On the other hand, mean values of MD showed no significant statistical difference between both patient and control groups. Other authors' findings showed decreased FA values. This may be owing to increased water diffusivity across neural fibre tracts, suggesting the existence of a dysmyelinating process owing to reduced functional activities along the auditory pathway, whereas MD values remained unchanged, denoting the absence of axonal injury or inflammation [7,14,18,28,37,38].

In our study, the changes in the FA values were higher at the level of the inferior colliculus than at the superior olivary nucleus and lateral lemniscus. This could be attributable to the distal anatomical location of the inferior colliculus in the central auditory circuit compared with the superior olive and the lateral lemniscus, and hence greater myelin degeneration because the inferior colliculus is a convergence site for inputs from different caudal auditory nuclei and hence may be more vulnerable to neuronal damage [14,28].

Because the auditory nervous system has early and multiple decussations starting from the level of the co- 
chlear nucleus $(\mathrm{CN})$, so it is expected that ipsilateral as well as contralateral auditory neural pathways could be affected by unilateral hearing loss with dominant affection in the contralateral side [29]. This was in agreement with our study, which showed reduced FA values in the ipsilateral side of affection when compared to controls in 4 patients with profound unilateral SNHL at the level of the lateral lemniscus and inferior colliculus, whereas the mean values of FA in the ipsilateral side at the 3 regions showed no statistical difference to those of the control group in other patients with unilateral SNHL.

Owing to previous results that reported the presence of an apparently linear relationship between declined FA values and audiometrically proven hearing loss, the FA may be considered as a valuable biological marker of the severity of hearing impairment $[7,14,25]$. In the same context, the current study showed that FA measures were inversely correlated with the level of hearing loss, whereas no correlation was noticed between MD and the level of hearing impairment.

Cochlear implantation is effective in the treatment of severe-to-profound SNHL [39]. However, there are still a remarkable number of patients with an unsatisfactory outcome after implantation. Therefore, finding a preoperative predictive tool is mandatory in the prognosis of postoperative outcome and in assisting surgical decisionmaking $[7,18]$.

Some studies have correlated fractional anisotropy values with audiometric tests after implantation surgery and found a significant positive correlation between categories of auditory performance scores and FA values. Other authors found that there were decreased FA values and unchanged MD values at the superior olivary nucleus, inferior colliculus, medial geniculate body, auditory radiation, and white matter of Heschl's gyrus in subjects with poor outcome when compared to subjects with good outcome. Thus, DTI of the auditory tract should be routinely done before implantation surgery $[18,25]$.
Finally, there were some limitations in our study. The main limitation was the small population in the SNHL group, hindering stratification of patients according to hearing loss severity. Second, young children were not included in this study, to avoid changes of FA due to myelin development and maturation, which is considered as a bias factor. In a healthy functioning auditory system, myelination begins in the early foetal life and extends projections to the primary auditory cortical centre, from 1 to 12 years old, and then the progression of axonal myelination tends to be stable [18].

Third, only 3 regions in the acoustic pathway were investigated in this study. Advance of tractography approaches may facilitate and improve discrimination of the less distinguishable cross-fibre regions of the auditory tract in the future [38]. Forth, there was no correlation between reduced FA values and outcomes in the cases indicated for implantation surgery. Some studies have mentioned that higher FA values at multiple regions along the auditory tract were noted in the good-outcome group of cochlear implantation, with a significant positive correlation between FA values and audiometric scores [7].

\section{Conclusions}

We conclude that a decline in FA values can be used to reflecting microstructural abnormalities of the central auditory tract in patients with idiopathic SNHL and well correlated with degree of hearing impairment. Information about central auditory pathway integrity should be included in MRI reports in patients who will undergo cochlear implantation.

\section{Conflict of interest}

The authors report no conflict of interest.

\section{References}

1. Lee CC, Sherman SM. On the classification of pathways in the auditory midbrain, thalamus, and cortex. Hear Res 2011; 276: 79-87.

2. Cunningham LL, Tucci DL. Hearing loss in adults. N Engl J Med 2017; 377: 2465-2473

3. Verbist BM. Imaging of sensorineural hearing loss: a pattern-based approach to diseases of the inner ear and cerebellopontine angle. Insights Imaging 2012; 3: 139-153.

4. Ouda L, Profant O, Syka J. Age-related changes in the central auditory system. Cell Tissue Res 2015; 361: 337-358.

5. Wu X, Chen K, Sun L, et al. Magnetic resonance imaging-detected inner ear hemorrhage as a potential cause of sudden sensorineural hearing loss. Am J Otolaryngol 2014; 35: 318-323.

6. Baird SM, Nguyen K, Bhatia DDS, Wei BPC. Inner ear and retrocochlear pathology on magnetic resonance imaging for sudden and pro- gressive asymmetrical sensorineural hearing loss. ANZ J Surg 2019; 89: 738-742.

7. Tarabichi O, Kozin ED, Kanumuri VV, et al. Diffusion tensor imaging of central auditory pathways in patients with sensorineural hearing loss: a systematic review. Otolaryngol Head Neck Surg 2018; 158: 432-442.

8. Sennaroglu L, Saatci I. A new classification for cochleovestibular malformations. Laryngoscope 2002; 112: 2230-2241.

9. Figueiredo EH, Borgonovi AF, Doring TM. Basic concepts of MR imaging, diffusion MR imaging, and diffusion tensor imaging. Magn Reson Imaging Clin N Am 2011; 19: 1-22.

10. Hasan KM, Walimuni IS, Abid H, Hahn KR. Review of diffusion tensor magnetic resonance imaging computational methods and software a tools. Comput Biol Med 2011; 41: 1062-1072. 
11. Nobuhara K, Okugawa G, Sugimoto T, et al. Frontal white matter anisotropy and symptom severity of late-life depression: a magnetic resonance diffusion tensor imaging study. J Neurol Neurosurg Psychiatry 2006; 77: 120-122.

12. Aung WY, Mar S, Benzinger TLS. Diffusion tensor MRI as a biomarker in axonal and myelin damage. Imaging Med 2013; 5: 427-440.

13. O’Donnell LJ, Westin CF. Introduction to diffusion tensor image analysis. Neurosurg Clin N Am 2011; 22: 185-196.

14. Chang Y, Lee SH, Lee YJ, et al. Auditory neural pathway evaluation on sensorineural hearing loss using diffusion tensor imaging. Neuroreport 2004; 15: 1699-1703.

15. Feldman HM, Lee ES, Yeatman JD, Yeom KW. Language and reading skills in school-aged children and adolescents born preterm are associated with white matter properties on diffusion tensor imaging. Neuropsychologia 2012; 50: 3348-3362.

16. Martin J, Endit S, Wetscherek A, et al. Contrast-to-noise ratio analysis of microscopic diffusion anisotropy inices in q-space trajectory. Z Med Phys 2020; 30: 4-16.

17. Kochunov P, Thompson PM, Lancaster JL, et al. Relationship between white matter fractional anisotropy and other indices of cerebral health in normal aging: tract-based spatial statistics study of aging. Neuroimage 2007; 35: 478-487.

18. Huang L, Zheng W, Wu C, et al. Diffusion tensor imaging of the auditory neural pathway for clinical outcome of cochlear implantation in pediatric congenital sensorineural hearing loss patients. PLoS One 2015; 10: 0140643

19. Kuhn M, Heman-Ackah SE, Shaikh JA, Roehm PC. Sudden sensorineural hearing loss a review of diagnosis, treatment, and prognosis. Trends Amplif 2011; 15: 91-105.

20. Chandrasekhar SS, Tsai BS, Schwartz SR, et al. Clinical practice guideline: sudden hearing loss (update) executive summary. Otolaryngol Head Neck Surg 2019; 161: 195-210.

21. Clyde JW, Patel VA, Kanekar S, Isildak H. Magnetic resonance imaging findings in idiopathic sudden sensorineural hearing loss. Acta Radiol 2019; 60: 1167-1174.

22. Yang $M$, Chen $H$, Liu B, et al. Brain structural and functional alterations in patients with unilateral hearing loss. Hearing Res 2014; 316: 37-43.

23. Hutson K, Durham D, Imig T, Tucci D. Consequences of unilateral hearing loss: cortical adjustment to unilateral deprivation. Hearing Res 2008; 237: 19-31.

24. Hutson KA, Durham D, Tucci DL. Consequences of unilateral hearing loss: time dependent regulation of protein synthesis in auditory brainstem nuclei. Hearing Res 2007; 233: 124-134.

25. Kim SY, Heo H, Kim DH, et al. Neural plastic changes in the subcortical auditory neural pathway after single-sided deafness in adult mice: a MEMRI study. Biomed Res Int 2018; 2018: 8624745.
26. Subramaniam K, Gill J, Fisher M, et al. White matter microstructure predicts cognitive training-induced improvements in attention and executive functioning in schizophrenia. Schizophr Res 2018; 193 : 276-283.

27. Aoki Y, Inokuchi R, Gunshin M, et al. Diffusion tensor imaging studies of mild traumatic brain injury: a meta-analysis. J Neurol Neurosurg Psychiatry 2012; 83: 870-876.

28. Lin Y, Wang J, Wu C, et al. Diffusion tensor imaging of the auditory pathway in sensorineural hearing loss: changes in radial diffusivity and diffusion anisotropy. J Magn Reson Imaging 2008; 28: 598-603.

29. Tae WS, Yakunina N, Kim TS, et al. Activation of auditory white matter tracts as revealed by functional magnetic resonance imaging. Neuroradiology 2014; 56: 597-605.

30. Peterson DC, Schofield BR. Projections from auditory cortex contact ascending pathways that originate in the superior olive and inferior colliculus. Hearing Res 2007; 232: 67-77.

31. Emmorey K, Allen JS, Bruss J, et al. A morphometric analysis of auditory brain regions in congenitally deaf adults. Proc Natl Acad Sci 2003; 100: 10049-10054.

32. Bengtsson SL, Nagy Z, Skare S, et al. Extensive piano practicing has regionally specific effects on white matter development. Nat Neurosci 2005; 8: 1148-1150.

33. Liu J, Dietz K, DeLoyht JM, et al. Impaired adult myelination in the prefrontal cortex of socially isolated mice. Nat Neurosci 2012; 15 : 1621-1623.

34. Schlegel AA, Rudelson JJ, Tse PU. White matter structure changes as adults learn a second language. J Cogn Neurosci 2012; 24: 16641670 .

35. Demerens C, Stankoff B, Logak M, et al. Induction of myelination in the central nervous system by electrical activity. Proc Natl Acad Sci U S A 1996; 93: 9887-9892.

36. Sinclair JL, Fischl MJ, Alexandrov O, et al. Sound-evoked activity influences myelination of brainstem axons in the trapezoid body. J Neurosci 2017; 37: 8239-8255.

37. Song SK, Sun SW, Ramsbottom MJ, et al. Dysmyelination revealed through MRI as increased radial (but unchanged axial) diffusion of water. Neuroimage 2002; 17: 1429-1436.

38. Jiang M, Wen Z, Long L, et al. Assessing cerebral white matter microstructure in children with congenital sensorineural hearing loss: a tract-based spatial statistics study. Front Neurosci 2019; 13: 597.

39. Moore DR, Shannon RV. Beyond cochlear implants: awakening the deafened brain. Nat Neurosci 2009; 12: 686-691. 